\title{
The role of a monoclonal antibody 11C8B1 as a diagnostic marker of IDH2-mutated sinonasal undifferentiated carcinoma
}

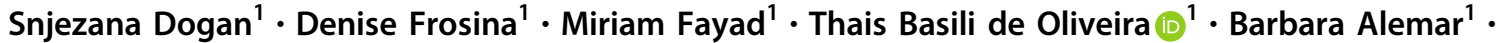 \\ Marc Rosenblum ${ }^{1} \cdot$ Laura H. Tang ${ }^{1} \cdot$ Meera Hameed $^{1} \cdot$ Bin Xu $\mathbb{C}^{2}{ }^{2}$. Ronald A. Ghossein ${ }^{1} \cdot$ Deborah J. Chute $^{3}$. \\ Britta Weigelt ${ }^{1}$ Achim A. Jungbluth ${ }^{1}$
}

Received: 9 June 2018 / Revised: 20 July 2018 / Accepted: 22 July 2018 / Published online: 11 September 2018

(c) United States \& Canadian Academy of Pathology 2018

\begin{abstract}
IDH2 R172 mutations occur in $>80 \%$ sinonasal undifferentiated carcinomas ("SNUC") and $~ 80 \%$ of these are R172S and R172T variants. We examined the utility of the monoclonal antibody 11C8B1 to IDH2 R172S in IDH2 R172-mutated tumors to establish an immunohistochemistry protocol as a surrogate method for $I D H 2$ R172S mutation detection. Eightyeight formalin-fixed paraffin-embedded tumors including 42 sinonasal tumors and a variety of IDH1/2-mutated malignancies were tested by immunohistochemistry. The IDHI/2 mutation status was determined in 86 cases by a targeted massively parallel sequencing MSK-IMPACT ${ }^{\mathrm{TM}}$ assay. Interestingly, monoclonal antibody 11C8B1 was reactive with all IDH2 R172S $(N=15)$ mutated tumors including 12 sinonasal carcinomas, 2 high-grade sarcomas and one intrahepatic cholangiocarcinoma, and with all R172T $(N=3)$ mutated sinonasal carcinomas displaying a distinct granular cytoplasmic labeling in all R172S/T mutated malignancies. 11C8B1 immunohistochemistry was also positive in 2 of 6 IDH1 R132S-mutated tumors, including one intrahepatic cholangiocarcinoma and one chondrosarcoma showing a smooth homogeneous cytoplasmic staining pattern. All IDH2 R172G/K/M/W $(N=22)$ and IDHI 132H/C/G/L $(N=15)$ mutated tumors, and all IDHI/2-wildtype tumors $(N=25)$, including a histologic variety of 23 sinonasal tumors, were immunonegative. Importantly, 11 sinonasal undifferentiated carcinomas $(N=14,79 \%)$ and $3(100 \%)$ high-grade neuroendocrine carcinomas, large cell type were 11C8B1 immunopositive. Literature search revealed a virtual absence of IDH2 R172 and IDHI R132S mutations in $>1000$ cases of 8 different malignancies included in the differential diagnosis of sinonasal undifferentiated carcinoma. Our study suggests that positive IDH2 11C8B1 immunohistochemistry in sinonasal carcinomas would be highly predictive of the presence of $I D H 2$ R172S/T mutations and could serve as a reliable adjunct diagnostic marker of sinonasal undifferentiated carcinomas in $>70 \%$ cases.
\end{abstract}

\section{Introduction}

IDH2 (isocitrate dehydrogenase 2) is a homodimeric mitochondrial enzyme that catalyzes the conversion of isocitrate to $\alpha$-ketoglutarate producing NADPH in the

Snjezana Dogan

dogans@mskcc.org

1 Department of Pathology, Memorial Sloan Kettering Cancer Center, New York, NY, USA

2 Department of Laboratory Medicine and Molecular Diagnostics, Sunnybrook Health Sciences Centre, Toronto, ON, Canada

3 Department of Pathology, Cleveland Clinic Foundation, Cleveland, $\mathrm{OH}$, USA process. The IDH2 R172 mutant protein gains a neomorphic ability to produce the "oncometabolite" 2hydroxyglutarate, which results in histone and DNA hypermethylation, and ultimately leads to block in cellular differentiation [1]. Over the past decade, activating somatic hotspot IDH1/2 mutations have been identified at variable proportions in various cancer types, including gliomas [2], chondrosarcomas [3], intrahepatic cholangiocarcinoma [4], acute myeloid leukemia [1, 5], angioimmunoblastic T-cell lymphoma [6], and solid papillary carcinoma with reverse polarity, a rare breast carcinoma subtype [7]. Given the relatively higher frequency, and the diagnostic and prognostic significance of IDH1 mutations in gliomas for example, the immunohistochemical detection of IDH1 R132H has been wellestablished and widely used in surgical pathology [8]. In 
Table 1 IDH2 11C8B1

immunohistochemistry in malignant tumors relative to their histologic type and $I D H 1 / 2$ mutation status (A), and in respect to the amino acid substitution (B)

\section{$\mathrm{A}$}

\begin{tabular}{|c|c|c|c|}
\hline $\begin{array}{l}\text { Tumor } \\
\text { category }\end{array}$ & Tumor type & $\begin{array}{l}I D H 2 \text { R } 172 \text { or } I D H 1 \\
\text { R132 mutation status } \\
(N)\end{array}$ & $\begin{array}{l}11 \mathrm{C} 8 \mathrm{~B} 1 \\
\text { (positive/ } \\
\text { total) }\end{array}$ \\
\hline \multirow{21}{*}{$\begin{array}{l}\text { Sinonasal } \\
\text { tumors } \\
(N=42)\end{array}$} & \multirow[t]{6}{*}{ Sinonasal undifferentiated carcinoma $(N=14)$} & R172S (9) & $9 / 9$ \\
\hline & & $\mathrm{R} 172 \mathrm{~T}(1)$ & $1 / 1$ \\
\hline & & R172M (1) & $0 / 1$ \\
\hline & & $\mathrm{R} 172 \mathrm{G}(1)$ & $0 / 1$ \\
\hline & & Wild-type (1) & $0 / 1$ \\
\hline & & Unknown (1) & $1 / 1$ \\
\hline & \multirow{2}{*}{$\begin{array}{l}\text { HG neuroendocrine carcinoma, large cell type } \\
(N=3)\end{array}$} & $\mathrm{R} 172 \mathrm{~S}(2)$ & $2 / 2$ \\
\hline & & $\mathrm{R} 172 \mathrm{~T}(1)$ & $1 / 1$ \\
\hline & \multirow{3}{*}{$\begin{array}{l}\text { PD non-intestinal type adenocarcinoma/carcinoma } \\
\text { with glandular/acinar differentiation }(N=7)\end{array}$} & $\mathrm{R} 172 \mathrm{~S}(1)$ & $1 / 1$ \\
\hline & & $\mathrm{R} 172 \mathrm{~T}(1)$ & $1 / 1$ \\
\hline & & Wild-type (5) & $0 / 5$ \\
\hline & MD intestinal type adenocarcinoma $(N=2)$ & Wild-type (2) & $0 / 2$ \\
\hline & Small cell neuroendocrine carcinoma $(N=2)$ & Wild-type (2) & $0 / 2$ \\
\hline & \multirow[t]{2}{*}{ SMARCB1-deficient carcinoma $(N=2)$} & Wild-type (1) & $0 / 1$ \\
\hline & & Unknown (1) & $0 / 1$ \\
\hline & $\begin{array}{l}\text { PD carcinoma with neuroendocrine and glandular } \\
\text { differentiation }(N=1)\end{array}$ & Wild-type (1) & $0 / 1$ \\
\hline & $\begin{array}{l}\text { HG neuroendocrine carcinoma with neuronal } \\
\text { differentiation }(N=1)\end{array}$ & Wild-type (1) & $0 / 1$ \\
\hline & Squamous cell carcinoma $(N=1)$ & Wild-type (1) & $0 / 1$ \\
\hline & $\begin{array}{l}\text { Combined small cell neuroendocrine carcinoma and } \\
\text { squamous cell carcinoma }(N=1)\end{array}$ & Wild-type (1) & $0 / 1$ \\
\hline & Olfactory neuroblastoma $(N=3)$ & Wild-type (3) & $0 / 3$ \\
\hline & Sinonasal melanoma $(N=5)$ & Wild-type (5) & $0 / 5$ \\
\hline \multirow{7}{*}{$\begin{array}{l}\mathrm{IHCC} \\
(N=9)\end{array}$} & \multirow[t]{7}{*}{ Intrahepatic cholangiocarcinoma $(N=9)$} & $\mathrm{R} 172 \mathrm{~S}(1)$ & $1 / 1$ \\
\hline & & R172W (2) & $0 / 2$ \\
\hline & & R172G (1) & $0 / 1$ \\
\hline & & R172M (1) & $0 / 1$ \\
\hline & & R132S (1) & $1 / 1$ \\
\hline & & $\mathrm{R} 132 \mathrm{G}(1)$ & $0 / 1$ \\
\hline & & Wild-type (2) & $0 / 2$ \\
\hline \multirow{6}{*}{$\begin{array}{l}\text { Sarcoma } \\
(N=6)\end{array}$} & HG multiphenotypic sarcoma $(N=1)$ & $\mathrm{R} 172 \mathrm{~S}(1)$ & $1 / 1$ \\
\hline & \multirow[t]{5}{*}{ HG chondrosarcoma $(N=5)$} & R172S (1) & $1 / 1$ \\
\hline & & $\mathrm{R} 172 \mathrm{G}(1)$ & $0 / 1$ \\
\hline & & R132S (1) & $1 / 1$ \\
\hline & & $\mathrm{R} 132 \mathrm{C}(1)$ & $0 / 1$ \\
\hline & & R132L (1) & $0 / 1$ \\
\hline \multirow{8}{*}{$\begin{array}{l}\text { Glioma } \\
(N=23)\end{array}$} & \multirow[t]{8}{*}{ All types $(N=23)$} & R172K (8) & $0 / 8$ \\
\hline & & $\mathrm{R} 172 \mathrm{~W}(2)$ & $0 / 2$ \\
\hline & & R172M (1) & $0 / 1$ \\
\hline & & $\mathrm{R} 172 \mathrm{G}$ (1) & $0 / 1$ \\
\hline & & R132S (4) & $0 / 4$ \\
\hline & & R132G (4) & $0 / 4$ \\
\hline & & R132H (2) & $0 / 2$ \\
\hline & & R132L (1) & $0 / 1$ \\
\hline
\end{tabular}


Table 1 (continued)

\begin{tabular}{|c|c|c|c|}
\hline \multicolumn{4}{|l|}{ A } \\
\hline $\begin{array}{l}\text { Tumor } \\
\text { category }\end{array}$ & Tumor type & $\begin{array}{l}I D H 2 \text { R172 or } I D H 1 \\
\text { R132 mutation status } \\
(N)\end{array}$ & $\begin{array}{l}\text { 11C8B1 } \\
\text { (positive/ } \\
\text { total) }\end{array}$ \\
\hline \multirow{3}{*}{$\begin{array}{l}\text { Lymphoma } \\
(N=3)\end{array}$} & Angioimmunoblastic T-cell lymphoma $(N=2)$ & $\mathrm{R} 172 \mathrm{M}(1)$ & $0 / 1$ \\
\hline & & $\mathrm{R} 172 \mathrm{G}(1)$ & $0 / 1$ \\
\hline & Peripheral T-cell lymphoma $(N=1)$ & $\mathrm{R} 172 \mathrm{G}(1)$ & $0 / 1$ \\
\hline \multirow{4}{*}{$\begin{array}{l}\text { Other tumors } \\
(N=5)\end{array}$} & Medullary thyroid carcinoma $(N=1)$ & $\mathrm{R} 132 \mathrm{~L}(1)$ & $0 / 1$ \\
\hline & Prostate adenocarcinoma $(N=1)$ & $\mathrm{R} 132 \mathrm{H}(1)$ & $0 / 1$ \\
\hline & Colorectal adenocarcinoma $(N=1)$ & $\mathrm{R} 132 \mathrm{C}(1)$ & $0 / 1$ \\
\hline & Endometrioid adenocarcinoma $(N=2)$ & $\mathrm{R} 132 \mathrm{C}(2)$ & $0 / 2$ \\
\hline \multicolumn{4}{|l|}{$\overline{\mathrm{B}}$} \\
\hline \multirow{12}{*}{\multicolumn{2}{|c|}{ All tumors with established mutation status $(N=86)$}} & R172S (15) & $15 / 15$ \\
\hline & & R172T (3) & $3 / 3$ \\
\hline & & R172K (8) & $0 / 8$ \\
\hline & & R172G (6) & $0 / 6$ \\
\hline & & R172W (4) & $0 / 4$ \\
\hline & & $\mathrm{R} 172 \mathrm{M}(4)$ & $0 / 4$ \\
\hline & & R132S (6) & $2 / 6$ \\
\hline & & $\mathrm{R} 132 \mathrm{G}(5)$ & $0 / 5$ \\
\hline & & R132C (4) & $0 / 4$ \\
\hline & & R132H (3) & $0 / 3$ \\
\hline & & R132L (3) & $0 / 3$ \\
\hline & & Wild-type (25) & $0 / 25$ \\
\hline
\end{tabular}

$H G$ high-grade, $P D$ poorly differentiated, $M D$ moderately differentiated, $I H C C$ intrahepatic cholangiocarcinoma

contrast, IDH2 mutations are less common, which may explain the lack of an established IDH2 immunohistochemistry protocols. Recently, we showed that the vast majority of sinonasal undifferentiated carcinomas ("SNUC", 82\%), and a variable proportion of other highgrade sinonasal epithelial malignancies, such as highgrade neuroendocrine carcinomas, large cell type, and poorly differentiated carcinomas with glandular/acinar differentiation, harbor somatic IDH2 R172 mutations with about $80 \%$ being either R172S or R172T variants [9]. Similar findings were also reported by Jo et al.; although, the frequency of $I D H 2$ mutant sinonasal undifferentiated carcinomas in their cohort of 11 cases was lower [10]. IDH2 single nucleotide variants are typically detected by DNA-based sequencing assays, which may be costly, time-consuming or simply unavailable in a pathology practice. An implementation of immunohistochemical assays targeting specifically IDH2 mutant proteins may provide a rapid, inexpensive alternate and/or corroborating method for mutant protein detection and help select cases amenable for further $I D H 2$ mutation confirmation. Except for a single study exploring the utility of a multi- specific antibody against IDH1/IDH2 mutant proteins in sinonasal tumors [11], monoclonal antibodies for specific detection of IDH2 R172S mutant protein have not been evaluated in formalin-fixed surgical specimens. In the present study, we examined the utility of a commercially available monoclonal antibody to IDH2 R172S in IDH2 R172-mutated tumors in order to establish an immunohistochemical protocol as a surrogate method for $I D H 2$ R172S mutation detection. In view of the very high recurrence rate of these mutations in sinonasal undifferentiated carcinomas in particular, we sought to explore potential advantages of IDH2 R172S immunohistochemistry as an adjunct diagnostic marker in this tumor type.

\section{Methods and materials}

\section{Cases}

A total of 88 formalin-fixed paraffin-embedded tumors were selected from the pathology archives (Memorial Sloan Kettering Cancer Center) for immunohistochemistry 

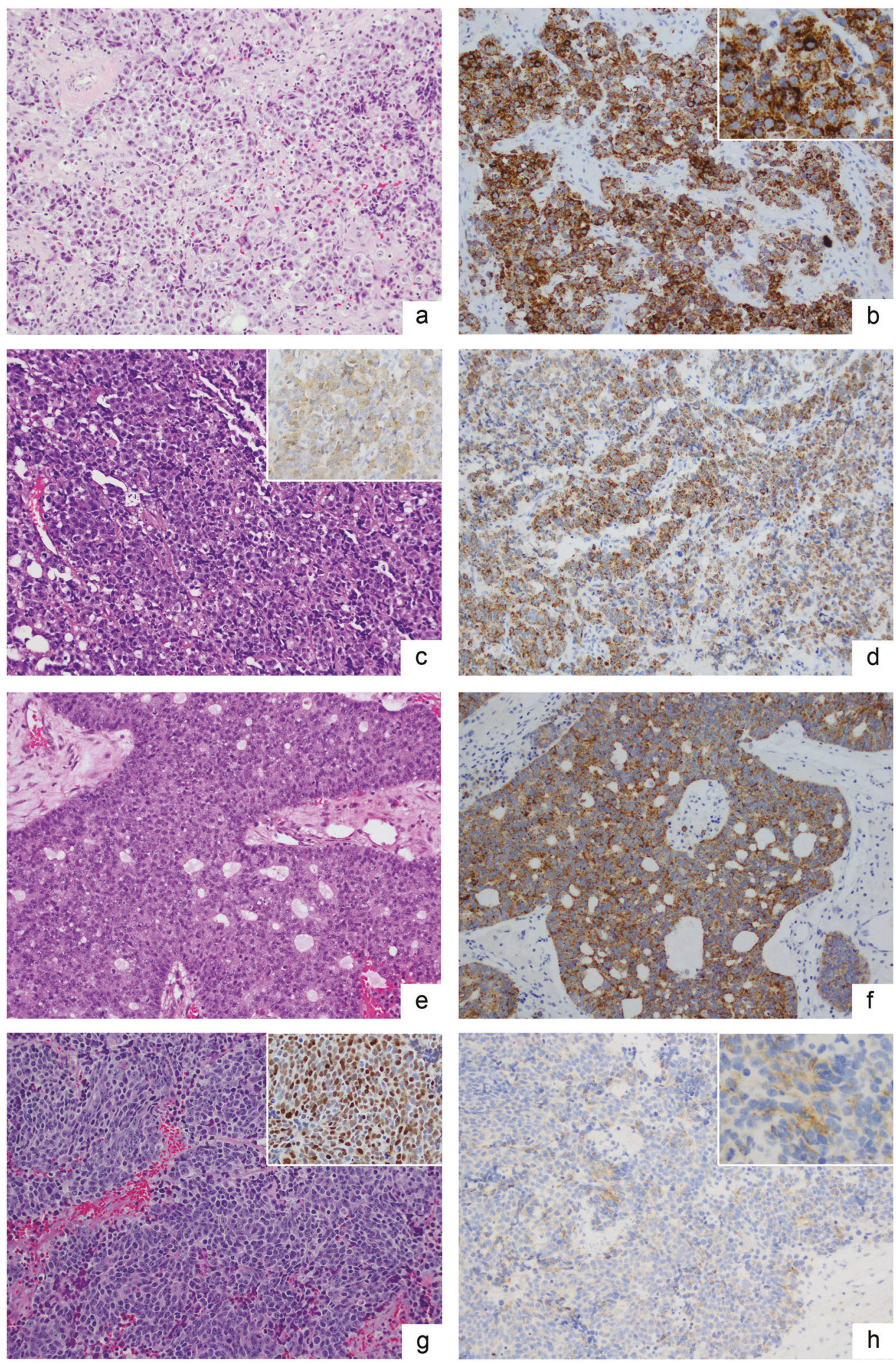

analysis. Upon approval by the Institutional Review Board, a combined research and clinical cohort of 42 sinonasal tumors, and a clinical cohort of selected IDHI/2-mutated non-sinonasal tumors with available material for testing $(N=46)$ were studied. The sinonasal cohort included 14 sinonasal undifferentiated carcinomas diagnosed using 
Fig. 1 IDH2 11C8B1 immunohistochemistry in sinonasal carcinomas. Sinonasal undifferentiated carcinoma harboring $I D H 2$ R172S variant comprised of sheets of large undifferentiated tumor cells with prominent nucleoli (a) shows granular cytoplasmic immunostaining pattern consistent with mitochondrial localization of IDH2 mutant protein as detected by 11C8B1 monoclonal antibody (b). High-grade neuroendocrine carcinoma, large cell type, IDH2 R172S mutant (c) demonstrates a diffuse positive immunolabeling for chromogranin (c, inset), and positive IDH2 immunohistochemistry (d). In the IDH2 R172T mutated poorly differentiated non-intestinal type adenocarcinoma (e) the immunostaining pattern was similar to that in R172S mutants (f). In one IDH2 R172T mutated high-grade neuroendocrine carcinoma, large cell type metastatic to liver (g), which labeled immunopositive for INSM1 (g, inset), the IDH2 immunohistochemistry was focal and granular $(\mathbf{h})$; $(\times 200$ magnification, insets $\mathbf{b}$ and h, $\times 400$ magnification)

the criteria as previously described [9, 12]. The clinical nonsinonasal cohort included gliomas $(N=23)$, intrahepatic cholangiocarcinoma $(N=9)$, chondrosarcoma $(N=5)$, angioimmunoblastic T-cell lymphoma $(N=2)$, endometrioid carcinoma $(N=2)$, and one of each: medullary thyroid carcinoma, colorectal adenocarcinoma, prostate adenocarcinoma, high-grade multiphenotypic sarcoma, and peripheral T-cell lymphoma (Table 1). The IDH1/2 mutation status was previously reported in 22 sinonasal carcinomas and 2 olfactory neuroblastomas [9]. Two sinonasal carcinomas had insufficient material for DNA extraction and the remaining 16 sinonasal tumors were profiled by our clinically validated FDA-approved Memorial Sloan Kettering-Integrated Mutation Profiling of Actionable Cancer Targets (MSK-IMPACT ${ }^{\mathrm{TM}}$ ) massive parallel sequencing assay targeting 341-468 cancer-related genes as previously described [13]. The $I D H 1 / 2$ mutation status in all non-sinonasal tumors was determined by MSK-IMPACT ${ }^{\mathrm{TM}}$ as a part of routine clinical work-up. In addition to the IDH2 immunohistochemistry performed on all cases as detailed below, other immunohistochemical studies depicted in the selected examples (Fig. 1c, g) were performed as a part of diagnostic work-up at the time of diagnosis.

\section{Immunohistochemistry protocol using IDH2 11C8B1 monoclonal antibody}

The monoclonal antibody, clone 11C8B1 (catalogue \#: 26408; NewEast Biosciences, Malvern, PA) generated to the target the IDH2 R172S mutant protein was obtained commercially. The clone designation (11C8B1) was not indicated on the accompanying specification sheet or website (http://www.neweastbio.com/) and was provided by the manufacturer upon request. For the original assessment of the immunohistochemical properties, we employed chondrosarcoma cell line SW1353, known to harbor IDH2 R172S mutation [14]. The authenticity of SW1353 was confirmed by short tandem repeats (STR) analysis (data not shown) and the presence of the mutation was confirmed by Sanger sequencing (data not shown). Cell pellets of SW1353 displayed consistent strong immunopositivity and sections thereof proved to be valuable positive controls and were also used for monoclonal antibody optimization. A panel of normal tissues was employed as negative controls and no immunostaining was observed in any of the concomitantly tested normal tissues. A variety of optimization tests were performed employing heat-based antigen retrieval as well as different retrieval buffers (citrate based low pH epitope retrieval buffer, ER1, Leica; EDTA-based high pH epitope retrieval buffer, ER2, Leica) and heating times between 15 and $30 \mathrm{~min}$ at the equipment set temperature of $99^{\circ} \mathrm{C}$. All immunohistochemical assays were performed on a Leica-Bond-3 automated stainer platform (Leica, Buffalo Grove, IL). The standard platform-associated polymeric detection kit (Refine, Leica) was used as secondary reagent.

\section{Results}

\section{C8B1 immunohistochemistry protocol}

11C8B1 worked best using a heat-based antigen retrieval method employing a high $\mathrm{pH}$ retrieval buffer (ER2, Leica) for $30 \mathrm{~min}$. A primary concentration of $0.5 \mu \mathrm{g} / \mathrm{ml}$ (1:2000) and $30 \mathrm{~min}$ incubation time resulted in a strong and consistent granular cytoplasmic staining. Once the conditions were optimized we used this protocol to test a pregenotyped cohort of $I D H 1 / 2$ mutated and $I D H 1 / 2$ wildtype tumors as outlined above for immunoreactivity with 11C8B1. Sections of SW1353 cell pellets were also used as positive controls in the consecutive steps of this study.

\section{Frequency of IDH2 11C8B1 immunopositivity in different tumor types}

Immunohistochemistry results are summarized in Table 1. Among the tumors with known IDH1/2 mutations status all IDH2 R172S mutant tumors (15/15, 100\%), including 12 sinonasal carcinomas (Fig. 1), one chondrosarcoma, one intrahepatic cholangiocarcinoma (Fig. 2), and one highgrade multiphenotypic sarcoma were immunohistochemistry positive. In addition, all three $I D H 2$ R172T-mutated sinonasal carcinomas $(3 / 3,100 \%)$ were immunopositive for 11C8B1. Interestingly, two out of six IDH1 R132S cases, including one intrahepatic cholangiocarcinoma, and one chondrosarcoma were also immunopositive although displayed a staining pattern distinct from that observed in IDH2 R172S/T mutant tumors (Fig. 3). All tumors harboring either IDH2 R172K/G/W/M $(N=22)$ or IDH1 132 $\mathrm{H} / \mathrm{G} / \mathrm{C} / \mathrm{L}(N=15)$ mutations, and all $I D H 1 / 2$ wild-type tumors $(N=25)$, including a histologic variety of 

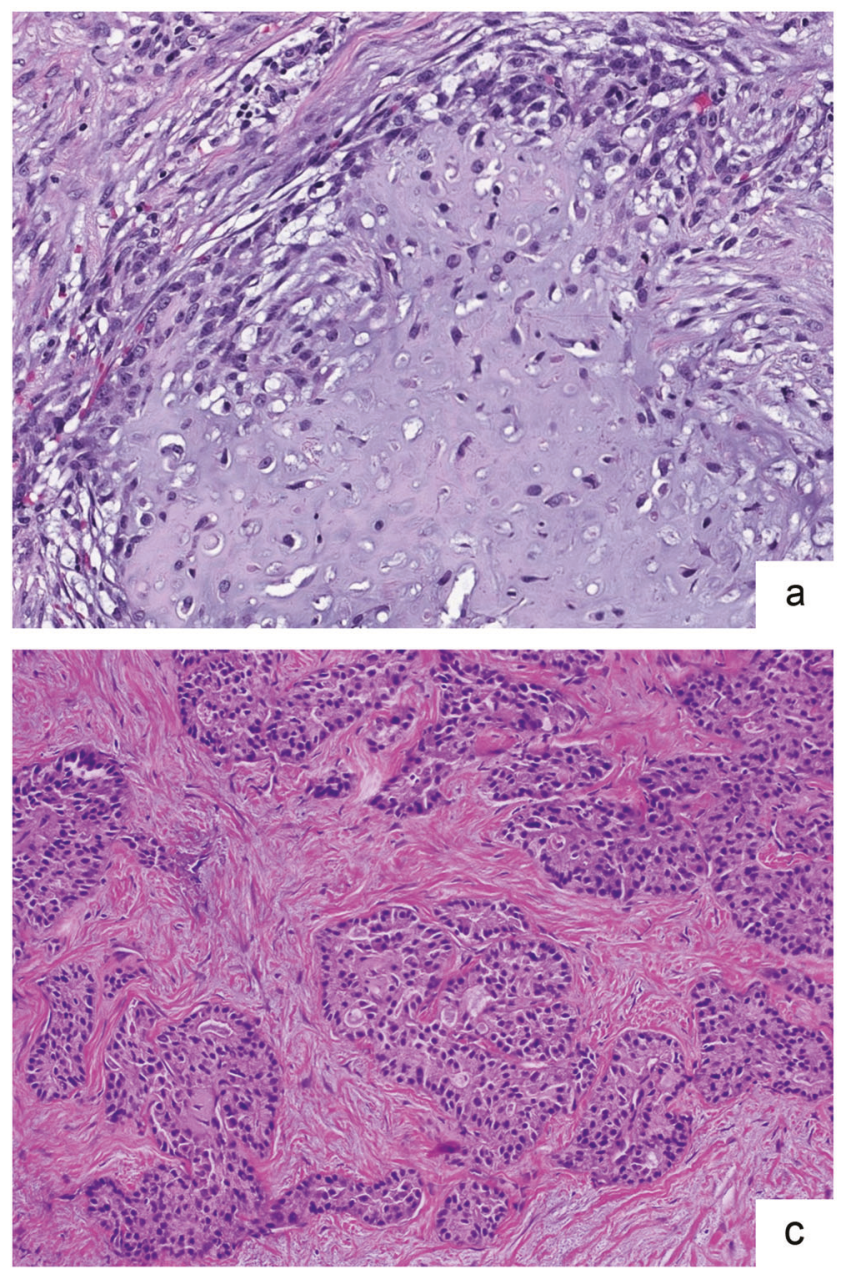

Fig. 2 IDH2 11C8B1 immunohistochemistry in IDH2 R172S mutated chondrosarcoma and intrahepatic cholangiocarcinoma. IDH2 R172Smutated chondrosarcoma (a, H\&E) displays cytoplasmic granular labeling for 11C8B1 monoclonal antibody (b), and intrahepatic

23 sinonasal tumors, were negative for $11 \mathrm{C} 8 \mathrm{~B} 1$ immunohistochemistry. One sinonasal undifferentiated carcinoma with unknown IDHI/2 mutation status was also positive for IDH2 immunohistochemistry and displayed granular cytoplasmic staining (Table 1B). Notably, among all sinonasal undifferentiated carcinomas irrespective of their $I D H I / 2$ mutation status, $11(N=14,79 \%)$ cases were positive for 11C8B1 immunohistochemistry, as well as all three $I D H 2$ R172S/T-mutated high-grade neuroendocrine carcinomas, large cell type.

\section{Immunohistochemical pattern of IDH2 11C8B1 staining}

The immunohistochemical stain in all IDH2 R172S/T mutated cases was diffuse and moderate to strong in all cases except for one IDH2 R172T-mutated high-grade neuroendocrine carcinomas, large cell type (Fig. 1). The

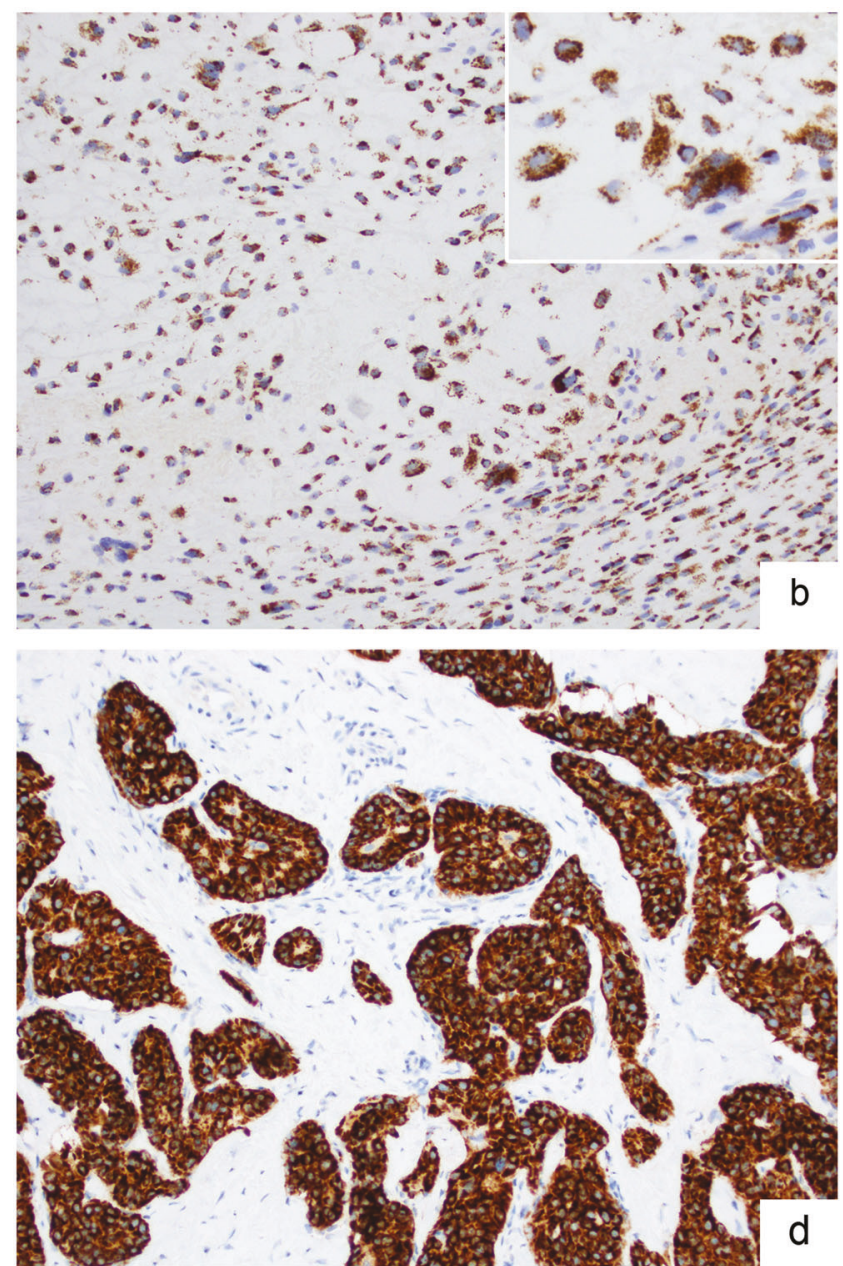

cholangiocarcinoma with the same mutation (c, H\&E) shows a similar yet very strong and diffuse immunostaining pattern (d, 11C8B1 immunohistochemistry); $\quad(\times 200$ magnification, inset $\times 400$ magnification)

latter case displayed only focal staining, but it was consistently present in the two tested specimens, in the primary tumor (not shown) and in the liver metastasis (Fig. 1h). Importantly, all IDH2 R172S/T-mutated malignancies showed strong granular cytoplasmic labeling, including sinonasal carcinomas (Fig. 1b, inset), as well as chondrosarcoma and intrahepatic cholangiocarcinoma (Fig. 2). In contrast, the two IDHI R132S-mutated tumors showed a homogeneous diffuse cytoplasmic staining lacking the distinct granularity observed in $I D H 2$ R172S/T-mutated cases (Fig. 3).

\section{Frequency of IDH2 R172S and R172T mutations in different tumor types}

Our immunohistochemistry results suggested that finding a positive immunostaining with monoclonal antibody 11C8B1 immunohistochemistry in a tumor without $I D H 2$ 


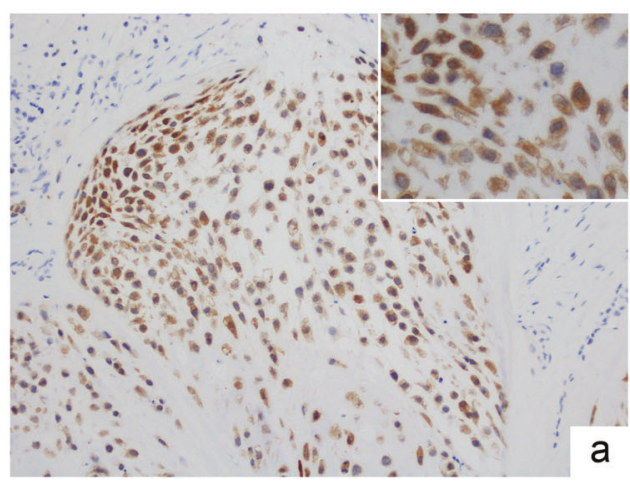

Fig. 3 IDH2 11C8B1 immunohistochemistry in IDH1 R132S-mutated malignancies. The staining pattern in IDH1 R132S-mutated chondrosarcoma was diffuse, moderately strong (a) showing a smooth homogeneous cytoplasmic distribution (a, inset). IDH1 R132S-

R172S/T or IDH1 R132S mutation would be very unlikely. This prompted us to search the literature to determine the frequency of these mutations in other tumors with emphasis on entities arising in the head and neck. Next-generation sequencing studies failed to reveal any $I D H 2$ mutation in $>1000$ cases among 8 relatively common entities that would be considered in the differential diagnosis of sinonasal undifferentiated carcinoma (Table 2), while an IDHI R132C variant occurred in a single case of adenoid cystic carcinoma. In addition, based on our own institutional experience reported in a pan-cancer study on $>10,000$ various predominantly solid malignancies profiled by MSKIMPACT $^{\mathrm{TM}}$ [15] $I D H 2$ hotspot variants occurred in only $0.3 \%$ cases with R172S/T mutations being detected in total $4(0.04 \%)$ cases including one of each; chondrosarcoma, angioimmunoblastic T-cell lymphoma, intrahepatic cholangiocarcinoma, and sinonasal undifferentiated carcinoma. IDHI hotspot mutations occurred in $2.5 \%$ cases including only $4(0.04 \%) \mathrm{R} 132 \mathrm{~S}$ variants detected in the tumors outside of the sinonasal tract (Table 2).

\section{Discussion}

Prompted by previous findings of highly recurrent $\mathrm{IDH} 2$ hotspot alterations, namely R172S/T variants in sinonasal undifferentiated carcinoma $[9,10]$, in the present study, we explored the feasibility and utility of an immunohistochemical assay in detecting the mutated IDH2 protein in standard archival surgical pathology specimens with a particular emphasis on potential diagnostic application of this immunostain in carcinomas of the sinonasal tract. We characterized the immunoreactivity of the monoclonal antibody $11 \mathrm{C} 8 \mathrm{~B} 1$ and demonstrated its usefulness as an immunohistochemical reagent for surgical pathology.

In order to validate our immunohistochemistry findings and to examine the specificity of $11 \mathrm{C} 8 \mathrm{~B} 1$, we took

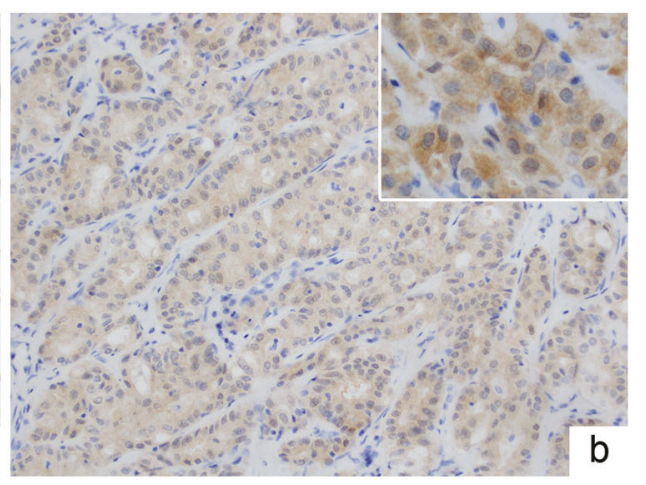

mutated intrahepatic cholangiocarcinoma was diffusely and weakly positive (b), and a homogeneous cytoplasmic labeling is appreciated even in the foci of stronger staining intensity (b, inset); ( $\times 200$ magnification, insets $\times 400$ magnification)

advantage of our pre-genotyped sinonasal tumors cohort and a large clinical cohort subjected to MSK-IMPACT ${ }^{\mathrm{TM}}$ mutational profiling to compile a histogenetically diverse control set comprising a variety of IDH1/2-mutated and wild-type tumors. Due to the high homology in the protein structure between the IDH2 and IDH1 mutation hotspot regions $\mathrm{R} 172$ and $\mathrm{R} 132$ respectively [16], testing of a variety of $I D H 1 \mathrm{R} 132$ mutants such as those having arginine $(\mathrm{R})$ substituted by cysteine $(\mathrm{C})$, histidine $(\mathrm{H})$, leucine $(\mathrm{L})$, glycine $(\mathrm{G})$, or serine $(\mathrm{S})$ was essential to explore potential cross-reactivity of the IDH2 11C8B1 antibody with IDH1 R132-mutated proteins.

11C8B1 showed consistent and strong granular cytoplasmic immunoreactivity with IDH2 R172S and IDH2 R172T mutant proteins consistent with mitochondrial localization of IDH2 protein [17]. It also showed weak to moderately strong homogeneous cytoplasmic immunoreactivity in the minority of IDHI R132S-mutated cases despite the manufacturer's designation this being a monoclonal antibody raised specifically against the IDH2 R172S protein. The cross-reactivity of the monoclonal antibody 11C8B1 between the R172S antibody and R172T mutant protein may be explained by a remarkable similarity in the chemical structure and properties between serine and threonine, which are both polar and neutral amino acids. However, from a practical perspective this relative lack in specificity has proven to be useful as we were able to detect all R172S/T variants by a single IDH2 monoclonal antibody. Similarly, given the high homology in the protein sequence between the IDH2 R172S and IDH1 R132S [16], the immunoreactivity of monoclonal antibody 11C8B1 observed in some IDH1 R132S mutants would not be unusual. The reasons for the lack of detection in other IDHI R132S-mutated cases remain unclear. Importantly, along with $I D H 2 \mathrm{R} 172 \mathrm{~K} / \mathrm{G} / \mathrm{W} / \mathrm{M}$ all remaining $I D H 1$ variants such as $\mathrm{R} 132 \mathrm{G} / \mathrm{C} / \mathrm{H} / \mathrm{L}$ were immunohistochemically negative for $11 \mathrm{C} 8 \mathrm{~B} 1$. We also showed that the $I D H 2$ 


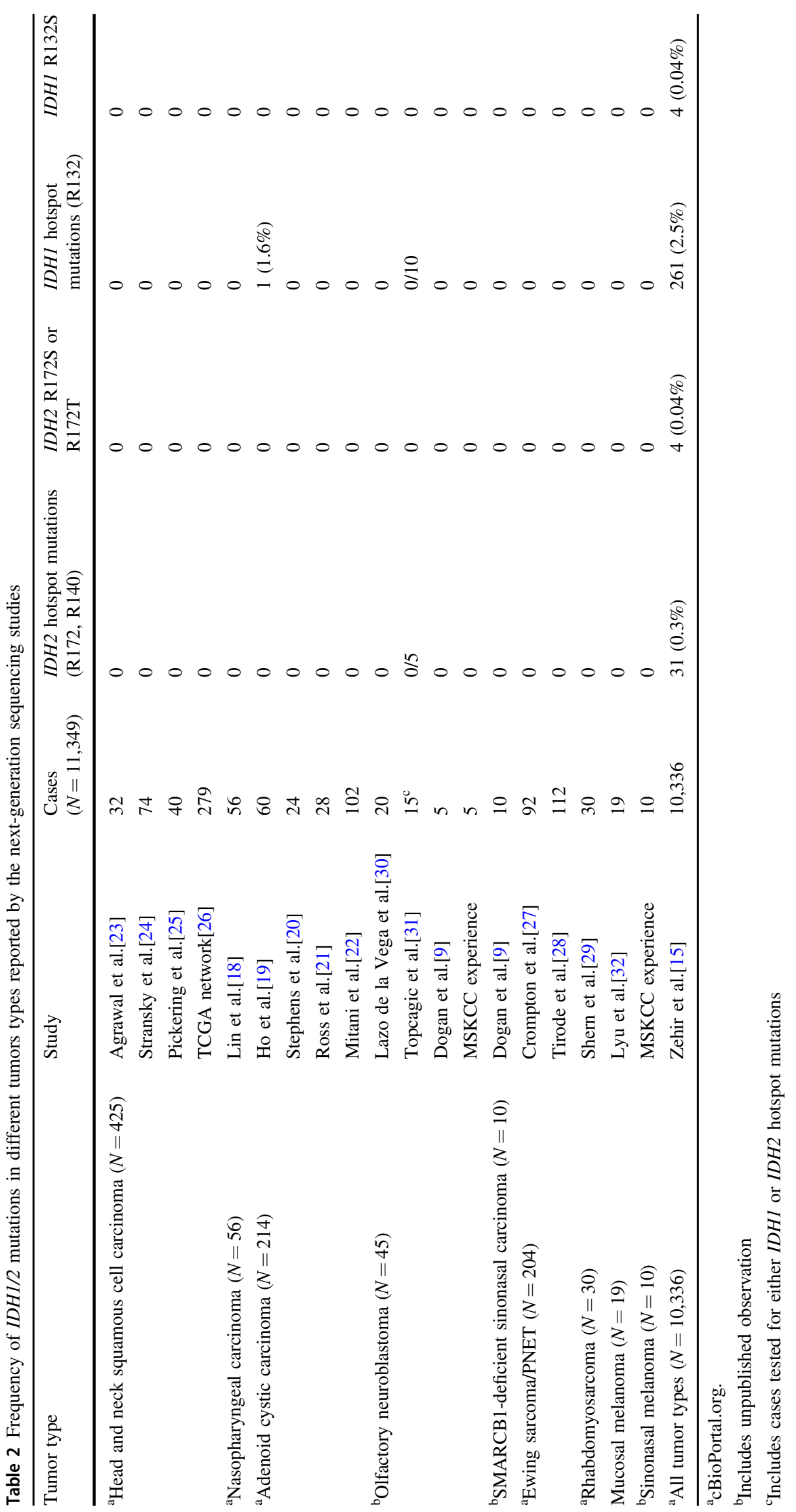


R172S-mutated SW1353 chondrosarcoma cell line pellets may serve as an inexhaustible positive control for IDH2 R172S immunohistochemistry, thus representing a suitable alternative to often limited clinical biopsy specimens.

Massively parallel sequencing studies have shown that, except for a single IDH1 R132C-mutated adenoid cystic carcinoma, other IDHI and IDH2 hotspot mutations are virtually absent in multiple tumor types that would typically be included in the differential diagnosis of sinonasal undifferentiated carcinoma. These include nasopharyngeal carcinoma [18], adenoid cystic carcinoma (solid type) [19-22], squamous cell carcinoma [23-26], Ewing sarcoma/PNET [27, 28], rhabdomyosarcoma (alveolar type) [29], olfactory neuroblastoma [9, 30, 31], and mucosal melanoma [32]. In addition, based on our limited experience, none of the 10 next-generation sequencing-profiled SMARCB1-deficient sinonasal carcinomas were found with a co-existing IDH2 or IDH1 variant [[9], including unpublished experience]. Similarly, in NUT carcinomas no other oncogenic mutations except for NUTM1 rearrangements have been identified to date [12].

Aside from sinonasal undifferentiated carcinoma, IDH2 R172S/T mutations may be highly recurrent in high-grade neuroendocrine carcinomas, large cell type as well. Taking into account one $I D H 2$-wild-type case we reported previously [9], in our experience, 3 of 4 (75\%) high-grade neuroendocrine carcinomas, large cell type harbored $\mathrm{IDH} 2$ $\mathrm{R} 172 \mathrm{~S} / \mathrm{T}$ variants. In contrast, no IDH2 mutation was detected in any of 3 sinonasal small cell neuroendocrine carcinomas, including one case reported previously [9]. The mutational profiles of the 2 sinonasal small cell neuroendocrine carcinomas demonstrated a remarkable genetic similarity to their lung counterpart [9]. Although such a small number of tested samples precludes any definitive conclusions, we speculate that the $I D H 2$ variants in sinonasal small cell neuroendocrine carcinoma might be as uncommon as in pulmonary small cell carcinomas. IDH2 mutations were not detected in any of 282 lung small cell carcinomas examined by a massive parallel sequencing [15, 33-36].

Based on the current evidence, a differential diagnosis of a high-grade carcinoma in the sinonasal tract that labels positive for IDH2 11C8B1 immunohistochemistry would include sinonasal undifferentiated carcinoma, high-grade neuroendocrine carcinomas, large cell type, and poorly differentiated non-intestinal type adenocarcinoma i.e. poorly differentiated carcinoma with focal glandular/acinar differentiation. An absence of bona fide neuroendocrine marker expression in a tumor without any evidence of any line of differentiation would be diagnostic of sinonasal undifferentiated carcinoma. Therefore, we suggest the IDH2 $11 \mathrm{C} 8 \mathrm{~B} 1$ immunohistochemistry should be added as one of the first line of immunostains in the diagnostic work-up of a poorly differentiated/undifferentiated sinonasal tumor. A more extensive diagnostic work-up, including costly ancillary studies such as in situ hybridization studies and/or molecular assays, may be considered only in IDH2 11C8B1 negative cases to rule out other entities, and/or to identify cases harboring less common variants such as IDH2 R172G or R172M.

In carcinomas outside of the sinonasal tract, a high frequency of IDH2 R172 variants was found in $77 \%$ solid papillary breast carcinomas with reverse polarity, a rare subtype characterized by a distinct histologic appearance resembling tall cell variant of papillary thyroid carcinoma [7]. In intrahepatic cholangiocarcinoma, IDH1 and IDH2 hotspot mutations occur in about $10 \%$ and $5 \%$ cases, respectively, and minority of those are R132S or R172S variants [4]. Therefore, the differential diagnosis of an IDH2 11C8B1 immunopositive metastatic/carcinoma of unknown primary would include sinonasal tract, biliary tract and breast in the top differential diagnosis. Distinct morphological features and relatively indolent biology of solid papillary breast carcinomas with reverse polarity, for example, may further help rule out this possibility [7]. Although IDH1 R132S and IDH2 R172S/T-mutated tumors cannot be distinguished based on the presence of $11 \mathrm{C} 8 \mathrm{~B} 1$ immunopositivity alone, a homogeneous cytoplasmic distribution i.e., a lack-of-distinct granularity in labeling may suggest IDH1 R132S over IDH2 R172S/T mutant.

Further on, IDH2 11C8B1 immunohistochemistry may have therapeutic implications in clinical practice since mutant IDH2 proteins represent an attractive therapeutic target. Clinical trials using selective IDH2-inhibitors for IDH2-mutated acute myeloid leukemia, for instance, have been in progress and promising results were recently published [37]. These findings suggest that a similar treatment approach may eventually be explored in other tumors harboring IDH2 mutations, including sinonasal carcinomas. In such cases, an addition of IDH2 immunohistochemistry in a diagnostic work-up could easily identify the cases amenable for further molecular IDH2 mutation confirmation and help select patients for clinical IDH2-inhibitors trials.

In conclusion, here we provide a first established immunohistochemical protocol for detection of mutant IDH2 using monoclonal antibody 11C8B1 in formalin-fixed paraffin-embedded surgical specimens. This monoclonal antibody detects IDH2 R172S/T proteins with optimal sensitivity irrespective of the tumor type. In view of the molecular epidemiology of the $I D H 2$ R172 variants across human malignancies and their notable predominance among high-grade sinonasal carcinomas, especially sinonasal undifferentiated carcinoma, we suggest that IDH2 11C8B1 immunohistochemistry could be used as a reliable surrogate marker for the presence of IDH2 R172S/T mutations in carcinomas in this location. Our study also provides an 
illustrative example of a molecular signature being translated into a powerful adjunct diagnostic marker with a potential to greatly enhance the diagnostic accuracy in pathologically challenging cases. In the diagnosis of sinonasal undifferentiated carcinoma, an entity that has been the subject of controversies and considered a diagnosis of exclusion for several decades since its first description in 1986 [38], this is significant progress.

Acknowledgements We wish to thank Dr. Frances Weis-Garcia and Antibody and Bioresource Core Facility (MSKCC) for providing the SW1353 cell line. We also thank Zoran Perak for his assistance in arranging the microphotographs. Research reported in this publication was supported by the Cancer Center Support Grant of the National Institutes of Health/National Cancer Institute under award number P30CA008748. B.A. was funded by CAPES (\#PDSE - 88881.133984/ 2016-01). The content is solely the responsibility of the authors and does not necessarily represent the official views of the National Institutes of Health.

\section{Compliance with ethical standards}

Conflict of interest The authors declare that they have no conflict of interest.

\section{References}

1. Marcucci G, Maharry $\mathrm{K}, \mathrm{Wu} \mathrm{YZ}$, et al. IDH1 and IDH2 gene mutations identify novel molecular subsets within de novo cytogenetically normal acute myeloid leukemia: a Cancer and Leukemia Group B study. J Clin Oncol. 2010;28:2348-55.

2. Yan H, Parsons DW, Jin G, et al. IDH1 and IDH2 mutations in gliomas. N Engl J Med. 2009;360:765-73.

3. Amary MF, Bacsi K, Maggiani F, et al. IDH1 and IDH2 mutations are frequent events in central chondrosarcoma and central and periosteal chondromas but not in other mesenchymal tumours. J Pathol. 2011;224:334-43.

4. Borger DR, Tanabe KK, Fan KC, et al. Frequent mutation of isocitrate dehydrogenase (IDH) 1 and IDH2 in cholangiocarcinoma identified through broad-based tumor genotyping. Oncology. 2012;17:72-9.

5. Mardis ER, Ding L, Dooling DJ, et al. Recurring mutations found by sequencing an acute myeloid leukemia genome. N Engl J Med. 2009;361:1058-66.

6. Cairns RA, Iqbal J, Lemonnier F, et al. IDH2 mutations are frequent in angioimmunoblastic T-cell lymphoma. Blood. 2012;119:1901-3.

7. Chiang S, Weigelt B, Wen H-C, et al. IDH2 mutations define a unique subtype of breast cancer with altered nuclear polarity. Cancer Res. 2016;76:7118-29.

8. Hartmann C, Hentschel B, Wick W, et al. Patients with IDH1 wild type anaplastic astrocytomas exhibit worse prognosis than IDH1mutated glioblastomas, and IDH1 mutation status accounts for the unfavorable prognostic effect of higher age: implications for classification of gliomas. Acta Neuropathol. 2010;120:707-18.

9. Dogan S, Chute DJ, Xu B, et al. Frequent IDH2 R172 mutations in undifferentiated and poorly-differentiated sinonasal carcinomas. J Pathol. 2017;242:400-8.

10. Jo VY, Chau NG, Hornick JL, et al. Recurrent IDH2 R172X mutations in sinonasal undifferentiated carcinoma. Mod Pathol. 2017;30:650-9.
11. Mito JK, Bishop JA, Sadow PM, et al. Immunohistochemical detection and molecular characterization of IDH-mutant sinonasal undifferentiated carcinomas. Am J Surg Pathol. 2018;42:1067-75.

12. El-Naggar AK, Chan JKC, Grandis JR, et al. eds. World Health Organization Classification of Head and Neck Tumours. 4th ed. Lyon, France: IARC Press; 2017. p. 18-21.

13. Cheng DT, Mitchell TN, Zehir A, et al. Memorial Sloan Kettering-Integrated Mutation Profiling of Actionable Cancer Targets (MSK-IMPACT): a hybridization capture-basednextgeneration sequencing clinical assay for solid tumor molecular onclogy. J Mol Diagn. 2015;17:251-64.

14. Barretina J, Caponigro G, Stransky N, et al. The Cancer Cell Line Encyclopedia enables predictive modelling of anticancer drug sensitivity. Nature. 2012;483:603-7.

15. Zehir A, Benayed R, Shah RH, et al. Mutational landscape of metastatic cancer revealed from prospective clinical sequencing of 10,000 patients. Nat Med. 2017;23:703-13.

16. Ward PS, Patel J, Wise DR, et al. The common feature of leukemia-associated IDH1 and IDH2 mutations is a neomorphic enzyme activity converting $\alpha$-ketoglutarate to 2 -hydroxyglutarate. Cancer Cell. 2010;17:225-34.

17. Yen K, Bittinger M, Su S, et al. Cancer-associated IDH mutations: biomarker and therapeutic opportunities. Oncogene. 2010;29:6409-17.

18. Lin D-C, Meng X, Hazawa M, et al. The genomic landscape of nasopharyngeal carcinoma. Nat Genet. 2014;46:866-71.

19. Ho AS, Kannan K, Roy DM, et al. The mutational landscape of adenoid cystic carcinoma. Nat Genet. 2013;45:791-8.

20. Stephens PJ, Davies HR, Mitani Y, et al. Whole exome sequencing of adenoid cystic carcinoma. J Clin Inves. 2013;123:2965-8.

21. Ross JS, Wang K, Rand JV, et al. Comprehensive genomic profiling of relapsed and metastatic adenoid cystic carcinomas by next-generation sequencing reveals potential new routes to targeted therapies. Am J Surg Pathol. 2014;38:235-8.

22. Mitani Y, Liu B, Rao PH, et al. Novel MYBL1 gene rearrangements with recurrent MYBL1-NFIB fusions in salivary adenoid cystic carcinomas lacking $\mathrm{t}(6 ; 9)$ translocations. Clin Cancer Res. 2016;22:725-33.

23. Agrawal N, Frederick MJ, Pickering CR, et al. Exome sequencing of head and neck squamous cell carcinoma reveals inactivating mutations in NOTCH1. Science. 2011;333:1154-7.

24. Stransky N, Egloff AM, Tward AD, et al. The mutational landscape of head and neck squamous cell carcinoma. Science. 2011;333:1157-60.

25. Pickering CR, Zhang J, Yoo SY, et al. Integrative genomic characterization of oral squamous cell carcinoma identifies frequent somatic drivers. Cancer Discov. 2013;3:770-81.

26. Network CGA. Comprehensive genomic characterization of head and neck squamous cell carcinomas. Nature. 2015;517:576-82.

27. Crompton BD, Stewart C, Taylor-Weiner A, et al. The genomic landscape of pediatric Ewing sarcoma. Cancer Discov. 2014:4:1326-41.

28. Tirode F, Surdez D, Ma X, et al. Genomic landscape of Ewing sarcoma defines an aggressive subtype with co-association of STAG2 and TP53 mutations. Cancer Discov. 2014;4:1342-53.

29. Shern JF, Chen L, Chmielecki J, et al. Comprehensive genomic analysis of rhabdomyosarcoma reveals a landscape of alterations affecting a common genetic axis in fusion-positive and fusionnegative tumors. Cancer Discov. 2014;4:216-31.

30. Lazo de la Vega L, McHugh JB, Cani AK, et al. Comprehensive molecular profiling of olfactory neuroblastoma identifies potentially targetable FGFR3 amplifications. Mol Cancer Res. 2017;15:1551-7.

31. Topcagic J, Feldman R, Ghazalpour A, et al. Comprehensive molecular profiling of advanced/metastatic olfactory neuroblastomas. PLoS ONE. 2018;13:e0191244. 
32. Lyu J, Song Z, Chen J, et al. Whole-exome sequencing of oral mucosal melanoma reveals mutational profile and therapeutic targets. J Pathol. 2018;244:358-66.

33. Peifer M, Fernández-Cuesta L, Sos ML, et al. Integrative genome analyses identify key somatic driver mutations of small-cell lung cancer. Nat Genet. 2012;44:1104-10.

34. Rudin CM, Durinck S, Stawiski EW, et al. Comprehensive genomic analysis identifies SOX2 as a frequently amplified gene in small-cell lung cancer. Nat Genet. 2012;44:1111-6.

35. George J, Lim JS, Jang SJ, et al. Comprehensive genomic profiles of small cell lung cancer. Nature. 2015;524:47-53.
36. Gardner EE, Lok BH, Schneeberger VE, et al. Chemosensitive relapse in small cell lung cancer proceeds through an EZH2SLFN11 axis. Cancer Cell. 2017;31:286-99.

37. Stein EM, DiNardo CD, Pollyea DA, et al. Enasidenib in mutant IDH2 relapsed or refractory acute myeloid leukemia. Blood. 2017;130:722-31.

38. Frierson HF, Mills SE, Fechner RE, et al. Sinonasal undifferentiated carcinoma. An aggressive neoplasm derived from the Schneiderian epithelium and distinct from olfactory neuroblastoma. Am J Surg Pathol. 1986;10:771-9. 